\title{
Influence of Impulse Moments On Single-Frequency Torsional Oscillations of Nonlinearly Elastic Bodies
}

\author{
Mariia Sokil \\ Lviv Polytechnic National University \\ Andriy Andrukhiv \\ Lviv Polytechnic National University \\ Solomiia Fedushko ( $\nabla$ solomiia.s.fedushko@lpnu.ua ) \\ Lviv Polytechnic National University \\ Natalia Kryvinska \\ Comenius University

\section{Yuriy Syerov} \\ Lviv Polytechnic National University \\ Maria Yurkiv \\ Lviv Polytechnic National University
}

\section{Research Article}

Keywords: oscillations, torsional, reliability, impulse, peculiarities

Posted Date: September 24th, 2021

DOI: https://doi.org/10.21203/rs.3.rs-923320/v1

License: (c) (i) This work is licensed under a Creative Commons Attribution 4.0 International License.

Read Full License 


\title{
scientific reports
}

\section{Influence of Impulse Moments on Single- Frequency Torsional Oscillations of Nonlinearly Elastic Bodies}

\author{
Mariia Sokil ${ }^{1}$, Andriy Andrukhiv ${ }^{1}$, Solomiia Fedushko ${ }^{1 *}$, Natalia Kryvinska ${ }^{2 *}$, Yuriy Syerov ${ }^{1}$, Maria Yurkiv ${ }^{1}$ \\ ${ }^{1}$ Lviv Polytechnic National University, Lviv, 79000, Ukraine \\ ${ }^{2}$ Comenius University in Bratislava, Bratislava, 820 05, Slovak Republic \\ * Corresponding authors: Solomiia Fedushko* (solomiia.s.fedushko@lpnu.ua).
}

\section{Abstract}

Analytical study of the impulse moment influences on the nonlinear torsional oscillations in the homogeneous constant cross-section of a body under classical boundary conditions of the first, second, and third types has been developed. For the case when the elastic material properties meet the body close to the power law of elasticity, mathematical models of the process are obtained. They are the boundary value problems for an equation of hyperbolic type with a small parameter at the discrete right-hand side. The latter expresses the effect of pulse momentum on the oscillatory process. The peculiarities of resonant oscillations are established. Relative torsional oscillations of a nonlinear elastic body that rotates around the axis with a constant portable angular velocity are considered, taking into account the periodic action of pulse momentum acting in a fixed cross-section. The reliability of the obtained calculation formulas is confirmed.

\section{Introduction}

The Introduction must include a review of the relevant literature that motivates the research question and a full description of the experimental aims (research questions) and hypotheses.

The creation and use of structural elements from new materials in various branches of the mechanical engineering and construction industry, the physical and mechanical properties of which differ from the classical correlations arising from the linear law of elasticity, require consideration of new mathematical models for evaluations of their elasticity efficiency. We are talking primarily about dynamic tension caused by deformations due to various external factors. If the dynamic deformations under continuous 
action on such elements for the case of simple stress state (torsion, tension, pure bending) can be obtained based on the mathematical models (torsional, longitudinal, bending) of oscillations for linear [1], quasilinear [2, 3] or partial strongly nonlinear $[4,5,6,7]$ laws of elasticity, for discrete action - the question for many cases remains open. The problem is related to the construction of analytical solutions of the corresponding boundary (mixed) problems for nonautonomous linear (quasilinear) equations of hyperbolic type. Numerical simulations of these mathematical models cannot provide generalized answers to many practical problems related to the discrete action of external loads. First, the resonance conditions and peculiarities of its passage under the periodic impulse of external perturbation. Such problems for the case of the elastic elements of structures under the condition of essentially (strongly) nonlinear law of its elastic properties of the consideration subject of work, whence the urgency of their consideration follows.

The most effective methods of investigating oscillating processes in nonlinear systems with concentrated masses and distributed parameters are those based on introductory provisions of perturbation methods $[2,8]$, combined with the principle of simultaneity of oscillations. A significant development was found when the set of single-frequency solutions can be built for undisturbed analogs of interconnected systems with the help of trigonometric and periodic Abet functions $[9,10]$. The latter describes the dynamics of a wide range of systems with power nonlinearity $[10,11,12]$ and has several features that are not typical for a linear system.

These are:

- First, the absence of the principle of superposition;

- Second, the oscillatory processes of these systems are characterized by features fundamentally different from linear systems as the dependence of the period of the dynamic process on the amplitude;

- Third, general analytical approaches to the study of perturbed analogs of systems are absent. All the above significantly complicates constructing an analytical solution of the corresponding mathematical models of the dynamic process under the action of impulse (discrete) load and obtaining on its basis correlations that would be the basis for engineering calculations. Thus, this work aims to develop a mathematical apparatus for studying the torsional oscillations of an elastic body whose elastic properties are described as close to the power law of elasticity [12] ( $\sigma=k \varepsilon^{v+l}$, where $\sigma$ is a tension in the body, $\varepsilon$ is a relative deformation, $v+1$ is a nonlinearity index, $v>-1$ and which is exposed to external impulse action.

\section{Methods}

The mathematical model of the dynamics of a nonlinear elastic body under the action of impulse moments.

It can be obtained without much difficulty based on the "dynamic equilibrium" of the conditionally selected element of the body. It is considered that its mass is evenly distributed along the length $l$, and the cross-section is constant. The deformation of such a body is unambiguously determined by its torsion $\phi(t, x)$ (the angle of torsion of the cross-section with the coordinate $x$ at any time) [3]. The latter is determined by torque in the specified cross-sections and the physical and mechanical properties of the material $\phi(t, x)=g\left(M_{k}\right)$. For the case considered in this work, $g\left(M_{k}\right)$ becomes $g\left(M_{k}\right)=G J_{P}\left(\frac{\partial \phi}{\partial x}\right)^{v+1}, \mathrm{G}$ is a "modulus of elasticity" of the second kind, and $J_{P}$ is the moment of inertia of the cross-section about the axis that passes through its geometric center. In this case, the ratio 
that stems from "dynamic equilibrium" for the element of the elastic body placed between crosssections $x$ and $x+d x$ is $\rho J_{p} \phi_{t t}=\frac{\partial}{\partial x}\left(G J_{P} \frac{\partial \phi}{\partial x}\right)^{v+1}$, where $\rho$ is the density of the body material. If, moreover, taking into account the small value of the resistance force $\varepsilon f\left(\phi_{t}, \phi_{x}, \phi_{x x}\right)$, the above ratio is transformed into the form of

$$
\phi_{t t}-\alpha^{2}\left(\phi_{x}\right)^{v} \phi_{x x}=\frac{\mu}{\rho} f\left(\phi_{t}, \phi_{x}, \phi_{x x}\right)
$$

In ratio (1) $\alpha^{2}=(v+1) \frac{G}{\rho}, \mu$ is a small parameter that shows that the maximum value of the righthand side equation (1) is a function $\frac{\mu}{\rho} f\left(\phi_{t}, \phi_{x}, \phi_{x x}\right)$ that is a small value compared to the maximum value of its left-hand side. In the case of the action on the elastic body at different times $t_{s}$. The momentum value of which generally depends on $\phi_{t}, \phi_{x}, \phi_{x x}$ equation (1) is transformed into the form of

$$
\phi_{t t}-\alpha^{2}\left(\phi_{x}\right)^{v} \phi_{x x}=\frac{\mu}{\rho}\left\{f\left(\phi_{t}, \phi_{x}, \phi_{x x}\right)+\sum_{s=1} h_{s}\left(\phi, \phi_{t}, \phi_{x}, \phi_{x x}\right) \delta\left(t-t_{s}\right) \delta\left(x-x_{s}\right)\right\}
$$

In (2), $\varepsilon h_{s}\left(\phi\left(t_{s}, x_{s}\right), \phi_{t}\left(t_{s}, x_{s}\right), \phi_{x}\left(t_{s}, x_{s}\right), \phi_{x x}\left(t_{s}, x_{s}\right)\right)$ is the magnitude of the momentum acting at time $t_{s}$ in the cross-section of the body with coordinate $x_{s}\left(0<x_{s}<l\right)$, and $\delta(\ldots)$ is a delta function of the corresponding argument [13]. The boundary conditions are classic: $1^{\text {st }}, 2^{\text {nd }}$ and $3^{\text {rd }}$ kind

$$
\begin{aligned}
& \phi(x, t)_{\mid x=0}=0, \phi(x, t)_{\mid x=l}=0, \\
& \phi(x, t)_{\mid x=0}=0, \phi_{x}(x, t)_{\mid x=l}=0, \\
& \phi_{x}(x, t)_{\mid x=0}=0, \phi_{x}(x, t)_{\mid x=l}=0,
\end{aligned}
$$

Corresponding to the absence of angles of torsion of the end of the elastic body - (3); free, unloaded ends - (4); and the absence of angle of torsion at the beginning of the body and free another end of the elastic body - (5).

It should be noted that boundary value problems formulated above also describe relative torsional oscillations of an elastic body that rotated around its axis of symmetry with a constant angular velocity $\Omega$ and $\phi(x, t)$, in such a case - the relative angle of torsion. From properties $\delta(\ldots)$ of the function, it is shown that the accuracy of the right-hand side of the differential equation (2), more precisely additive components that describe impulse action on the elastic body, will not change if presented as

$$
\begin{aligned}
& \sum_{s=1} h_{s}\left(\phi, \phi_{t}, \phi_{x}, \phi_{x x}\right) \delta\left(t-t_{s}\right) \delta\left(x-x_{s}\right)=\sum_{s=1} h_{s}\left(\phi, \phi_{t}, \phi_{x}, \phi_{x x}\right) \cos \theta_{s} \delta\left(\frac{\theta-\bar{\theta}_{s}}{\Theta_{s}}\right) \delta\left(x-x_{s}\right), \\
& \theta_{s}=\Theta_{s} t, \bar{\theta}_{s}=\Theta_{s} t_{s}, \Theta_{s}=\frac{2 \pi}{t_{s+1}-t_{s}}
\end{aligned}
$$

This allows us to consider differential equations formally 


$$
\phi_{t t}-\alpha^{2}\left(\phi_{x}\right)^{v} \phi_{x x}=\frac{\mu}{\rho}\left\{f\left(\phi_{t}, \phi_{x}, \phi_{x x}\right)+\sum_{s=1} \mathrm{~h}_{s}\left(\phi, \phi_{t}, \phi_{x}, \phi_{x x}\right) \cos \theta_{s} \delta\left(\frac{\theta_{s}-\bar{\theta}_{s}}{\Theta_{s}}\right) \delta\left(x-x_{s}\right)\right\}
$$

nonautonomous, and the nonautonomous part is proportional to the small parameter. Many works [2, $14,15]$ show that in a nonresonant case, a small periodic perturbation of the nonautonomous type for the first approximation does not affect the basic parameters of the dynamic process. Therefore, only the case of periodic action of impulse perturbation, i.e., $t_{s+1}-t_{s}=\tau=$ const, $\mathrm{s}=1,2, \ldots$ will be considered below. In this case, the differential equations (7) can be written as

$$
\phi_{t t}-\alpha^{2}\left(\phi_{x}\right)^{v} \phi_{x x}=\frac{\mu}{\rho}\left\{f\left(\phi_{t}, \phi_{x}, \phi_{x x}\right)+\sum_{s=1} \mathrm{~h}_{s}\left(\phi, \phi_{t}, \phi_{x}, \phi_{x x}\right) \cos \theta \delta\left(\frac{\theta-2 \pi(s-1)}{\tau}\right) \delta\left(x-x_{s}\right)\right\}
$$

\section{Results}

Nonresonant case.

Using the general ideas of asymptotic methods of nonlinear mechanics adapted for boundary value problems for equation (1) in [14], the first approximation of the asymptotic solution of equation (8) in the form close to $\mathrm{k}$ that form of dynamic equilibrium is presented in the form

$$
\phi(x, t)=a_{k}(t) \Phi_{k}(x) c a\left(v+1,1, \psi_{k}\right)+\mu U_{1 k}\left(a_{k}, x, \psi_{k}, \theta\right), \psi_{k}(t)=\omega_{k}\left(a_{k}\right) t+\vartheta_{k}
$$

where $a_{k}(t)$ and $\psi_{k}(t)$ are the amplitude and phase of a single-frequency dynamic process of an elastic body, $\Phi_{k}(x)$ respectively, and $\omega_{k}(a)$ and are a system of functions that describes the forms of oscillations of undisturbed motion and its frequency, respectively.

They are expressed for boundary conditions (3) - (5) through periodic Ateb functions, respectively, in the form

$$
\begin{aligned}
& \left\{\Phi_{k}(x)\right\}=\left\{\begin{array}{l}
s a\left(1, \frac{1}{v+1}, \frac{k}{l} \Pi_{x} x\right), \\
c a\left(1, \frac{1}{v+1} \frac{k}{l} \Pi_{x} x\right), \\
s a\left(1, \frac{1}{v+1}, \frac{2 k+1}{2 l} \Pi_{x} x\right)
\end{array}\right\} \\
& \omega_{k}\left(a_{k}\right)=\left\{\begin{array}{l}
\alpha\left(\frac{k \Pi_{x}}{l}\right)^{1+\frac{v}{2}} a_{k}^{\frac{v}{2}}, \\
\alpha\left(\frac{k \Pi_{x}}{l}\right)^{1+\frac{v}{2}} a_{k}^{\frac{v}{2}}, \\
\alpha\left(\frac{(2 k+1) \Pi_{x}}{2 l}\right)^{1+\frac{v}{2}} a_{k}^{\frac{v}{2}},
\end{array}\right.
\end{aligned}
$$


$\Pi_{x}=\sqrt{\pi} \Gamma\left(\frac{v+1}{v+2}\right)\left(\Gamma\left(\frac{1}{2}+\frac{v+1}{v+2}\right)\right)^{-1}$ is a half-period of Abet functions used, which describes the

forms of oscillations.

For the function $U_{1 k}\left(a_{k}, x, \psi_{k}, \theta\right)$, which considers the influence of small impulse forces and dissipative forces, it must be periodic by arguments $\psi_{k}$ and $\theta$, by an argument $\psi_{k}$ with a period that is equal to $2 \Pi=2 \sqrt{\pi} \Gamma\left(\frac{1}{v+2}\right)\left(\Gamma\left(\frac{1}{2}+\frac{1}{v+2}\right)\right)^{-1}$, and by arguments $\theta_{-} 2 \pi$ is periodic. In addition, it should not contain $k^{\text {th }}$ modes of natural oscillations of undisturbed motion. The later is equivalent to the following

$$
\int_{0}^{2 \Pi_{\psi}} U_{1 k}\left(a_{k}, x, \psi_{k}, \theta\right)\left\{\begin{array}{l}
c a\left(v+1,1, \psi_{k}\right) \\
s a\left(1, v+1, \psi_{k}\right)
\end{array}\right\} d \psi_{k}=0
$$

The physical interpretation imposed on the function is the following: the amplitude of the $k$ - mode of the dynamic process is accepted as the amplitude of single-frequency torsional oscillations of the elastic body. In addition, this function must satisfy the boundary conditions that are consistent with (3) - (5), i.e.,

$$
\begin{aligned}
& U_{1 k}\left(a_{k}, x, \psi_{k}, \theta\right)_{\mid x=0}=0, U_{1 k}\left(a_{k}, x, \psi_{k}, \theta\right)_{\mid x=l}=0 \\
& {\frac{U_{1 k}\left(a_{k}, x, \psi_{k}, \theta\right)}{\partial x}}_{\mid x=0}=0,{\frac{U_{1 k}\left(a_{k}, x, \psi_{k}, \theta\right)}{\partial x}}_{|x=0| x=l}=0 \\
& U_{1 k}\left(a_{k}, x, \psi_{k}, \theta\right)_{\mid x=0}=0,{\frac{U_{1 k}\left(a_{k}, x, \psi_{k}, \theta\right)}{\partial x}}_{|x=0| x=l}=0
\end{aligned}
$$

In figure 1, dependencies (11) for the boundary conditions (3) or (4) change of the natural frequency of the oscillations due to amplitude and the parameter of nonlinearity, respectively, and in figure 1c) and 1d) are the dependence of the natural frequency of oscillations on the parameter $\alpha$ and the amplitude of oscillations at fixed values of the nonlinearity parameter. For the law of change of the frequency of natural nonlinear oscillations under boundary conditions (5), the qualitative picture of their change does not change, and the quantitative picture takes on slightly different values due to a different value of the parameter at the coefficient $\alpha$.

a) $l=2, \alpha=500, n=v$, axis $-\omega_{1}\left(a_{1}\right)$

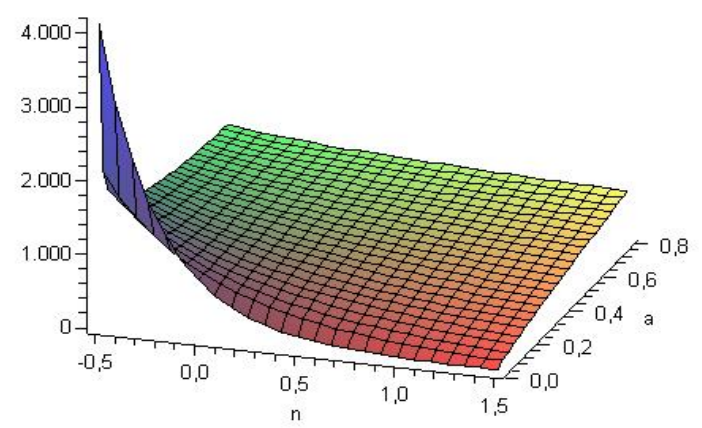

b) $l=4, \alpha=500, n=v$, axis $-\omega_{1}\left(a_{1}\right)$

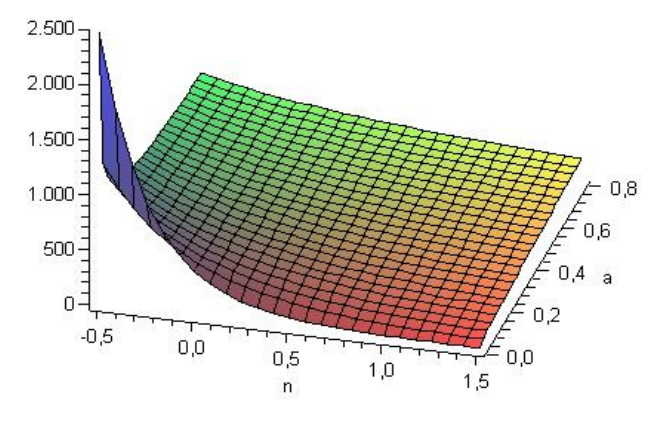


c) $v=-03,300,<\alpha<1000$, axis $-\omega_{1}\left(a_{1}\right), L-\alpha$

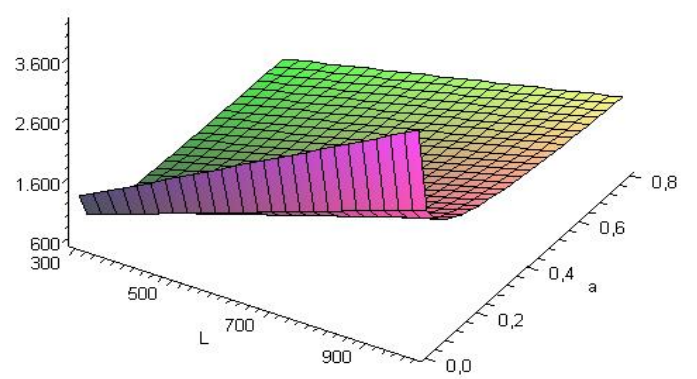

d) $v=03,300,<\alpha<1000$, axis $-\omega_{1}\left(a_{1}\right), L-\alpha$

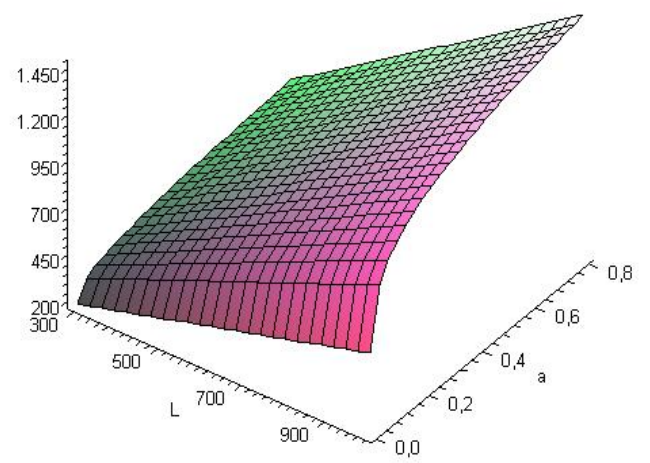

Figure 1: Dependence of the natural frequency of torsional oscillations of a nonlinear elastic body on the amplitude of oscillations and other characteristics of the body

The presented graphical dependences show:

- for more significant values of nonlinearity parameter $v$ (for all other invariant characteristics of elastic body), natural frequency of torsional oscillations is lower;

- the rate of decrease of the amplitude depending on the parameter $v$ is more significant for its smaller values;

- for greater values of the amplitude of the oscillations in case $-1<v<0$ the natural frequency decreases, and in the case $v>0$ is an increase;

- with an increased coefficient of "stiffness" $\alpha$ at $v>0$ natural frequency increases (for all other constant parameters of elastic body), and $-1<v<0$ is a decrease.

If we substitute in the differential equation (7) in place of the unknown function $\phi(x, t)$ and its derivatives the correlations arising from the representation of the asymptotic solution in the form (8), then after equalizing the coefficients in the right and left parts of the obtained expression at small parameter $\mu$ we obtain linear differential equation which connects the desired functions $a_{k}(t)$ and $\psi_{k}(t)$, more precisely their time derivatives $\frac{d a_{k}}{d t}, \frac{d \vartheta_{k}}{d t}$ function $U_{1 k}\left(a_{k}, x, \psi_{k}, \theta\right)$ and operation moments as

$$
\begin{aligned}
& \frac{\partial^{2} U_{1 k}}{\partial \psi_{k}^{2}} \omega_{k}^{2}\left(a_{k}\right)+\frac{\partial^{2} U_{1 k}}{\partial \theta^{2}} \mu^{2}+2 \frac{\partial^{2} U_{1 k}}{\partial \theta \partial \psi_{k}} \mu \omega_{k}\left(a_{k}\right)- \\
& -\alpha^{2}\left(a_{k} c a\left(v+1,1, \psi_{k}\right)\right)^{v}\left\{\left(\frac{d \Phi_{k}(x)}{d x}\right)^{v} \frac{\partial^{2} U_{1 k}}{\partial x^{2}}+v\left(\frac{d \Phi_{k}(x)}{d x}\right)^{v-1} \frac{d^{2} \Phi_{k}(x)}{d x^{2}} \frac{\partial U_{1 k}}{\partial x}\right\}= \\
& =\frac{2}{(v+2)}\left[\frac{d a_{k}}{d t}\left(\omega_{k}\left(a_{k}\right)+a_{k} \frac{d \omega_{k}}{d a_{k}}\right) s a\left(1, v+1, \psi_{k}\right)+a_{k} \omega_{k}\left(a_{k}\right) \frac{d \vartheta}{d t} c a^{v+1}\left(v+1,1, \psi_{k}\right)\right] \Phi_{k}(x)+ \\
& +\rho^{-1} F_{1 k}\left(a_{k}, x, \psi_{k}\right)+\rho^{-1} \sum_{s=1} G_{1 s}\left(a_{k}, x, \psi_{k}\right) \cos \theta \delta\left(\frac{\theta}{\tau}-\frac{2(s-1) \pi}{\tau}\right) \delta\left(x-x_{s}\right),
\end{aligned}
$$


where $F_{l k}\left(a_{k}, x, \psi_{k}\right)$ and $G_{1 s}\left(a_{k}, x, \psi_{k}\right)$ correspond to the values of functions $f\left(\phi, \phi_{t}, \phi_{x}, \phi_{x x}\right)$ and $g_{s}\left(\phi, \phi_{t}, \phi_{x}, \phi_{x x}\right)$ provided that they $\phi(x, t)$ and their derivatives are determined according to the principal value of a specified function $\phi(x, t)$, i.e., $\phi(x, t)=a_{k} \Phi_{k}(x) c a\left(v+1,1, \psi_{k}\right)$.

Thus, the problem for the first asymptotic approximation is to find such correlations for the unknown functions $a_{k}(t)$ and $\psi_{k}(t)$, which together with (9) and (16) satisfy the initial equation (8) with the considered accuracy. To solve this problem to some extent will allow the properties of completeness and orthonormality of the system of functions that describe the shape of oscillations, i.e.

$$
\int_{0}^{l} \Phi_{p}(x) \Phi_{q}(x) d x=\left\{\begin{array}{l}
0 \text { np } \mathrm{p} \neq \mathrm{q} \\
\Delta \text { nрu } p=q
\end{array}\right.
$$

$\Delta=\frac{v+2}{3 v+4} l$ for boundary conditions (13) and (14) and $\Delta=\frac{v+2}{3 v+4} \frac{l}{2}$ - for boundary conditions (15).

The specified property of the system of functions $\{\Phi(x)\}$ allows:

- delta function on the linear variable to be presented in the form

$$
\delta\left(x-x_{s}\right)=\frac{1}{\Delta} \sum_{j=1} \Phi_{j}\left(x_{s}\right) \Phi_{j}(x)
$$

that is, to avoid sampling the basic equation by a linear variable;

- unknown functions $U_{1 k}\left(a_{k}, x, \psi_{k}, \theta\right)$ in the asymptotic representation of a single-frequency solution are presented in the form

$$
U_{l k}\left(a_{k}, x, \psi_{k}, \theta\right)=\sum_{\substack{m=1 \\ m \neq k}} \Phi_{m}(x) U_{l k m}\left(a_{k}, \psi_{k}, \theta\right)
$$

the boundary conditions, in this case, will be fulfilled automatically.

If function $U_{1 k}\left(a_{k}, x, \psi_{k}, \theta\right)$ is $2 \Pi$ - periodic by argument $\psi_{k}$ and is not contained in the schedule of the first harmonic $\psi_{k}$, its derivatives have the same property. Therefore, by substituting the correlations (18) and (19) into the differential equation (16), at the same time using the properties of the system of functions $\{\Phi(x)\}$, simple differential equations that describe the change in the amplitude of time and phase of the single-frequency process of torsional oscillations of the elastic body can be found from the obtained expression, as well as equations in partial derivatives that connect unknown coefficients $U_{l k m}\left(a_{k}, \psi_{k}, \theta\right)$ of the decomposition of the function $U_{l k}\left(a_{k}, x, \psi_{k}, \theta\right)$, in the form of 


$$
\begin{aligned}
& \frac{d a_{k}}{d t}=-\frac{\mu s a\left(1, v+1, \psi_{k}\right)}{2 \omega_{k}\left(a_{k}\right) \rho \Delta} \int_{0}^{l}\left\{\begin{array}{l}
F_{1 k}\left(a_{k}, x, \psi_{k}\right)+ \\
\left.+\sum_{s=1} G_{1 s}\left(a_{k}, x, \psi_{k}\right) \cos \theta \delta\left(\frac{\theta}{\tau}-\frac{2(s-1) \pi}{\tau}\right)\right\} \Phi_{k}(x) d x \\
\sum_{j=1} \Phi_{j}\left(x_{s}\right) \Phi_{j}(x)
\end{array}\right] \\
& \frac{d \psi_{k}}{d t}=\omega_{k}\left(a_{k}\right)- \\
& -\frac{\mu(v+2) c a^{v+1}\left(v+1,1, \psi_{k}\right)}{2 a_{k} \omega_{k}\left(a_{k}\right) \rho \Delta} \int_{0}^{l}\left\{\begin{array}{l}
F_{1 k}(a, x, \psi)+ \\
\Delta \sum_{s=1} G_{1 s}\left(a_{k}, x, \psi_{k}\right) \cos \theta \delta\left(\frac{\theta}{\tau}-\frac{2(s-1) \pi}{\tau}\right) \Phi_{k}(x) d x \\
\sum_{j=1} \Phi_{j}\left(x_{s}\right) \Phi_{j}(x)
\end{array}\right\} \\
& \frac{\partial^{2} U_{1 k m}\left(a_{k}, \psi_{k}, \theta\right)}{\partial \psi_{k}^{2}} \omega_{k}^{2}\left(a_{1 k}\right)+\frac{\partial^{2} U_{1 k m\left(a_{k}, \psi_{k}, \theta\right)} \mu^{2}+}{\partial \theta^{2}} \\
& +2 \frac{\partial^{2} U_{1 k m}\left(a_{k}, \psi_{k}, \theta\right)}{\partial \theta \partial \psi_{k}} \mu \omega_{k}\left(a_{k}\right)-\bar{\alpha}_{k m}^{2}\left(a_{k}, \psi_{k}\right) U_{1 k m}\left(a_{k}, \psi_{k}, \theta\right)= \\
& \rho^{-1} F_{1 k m}\left(a_{k}, \psi_{k}\right)+(\rho \Delta)^{-1} \sum_{s=1} G_{1 s m}\left(a_{k}, \psi_{k}\right) \cos \theta \delta\left(\frac{\theta}{\tau}-\frac{2(s-1) \pi}{\tau}\right) \sum_{j=1} \Phi_{j}\left(x_{s}\right) \Phi_{j}(x)
\end{aligned}
$$

where $F_{1 k m}\left(a_{k}, \psi_{k}\right)=\frac{1}{\Delta} \int_{0}^{l} F_{1 k}\left(a_{k}, x, \psi_{k}\right) \Phi_{m}(x) d x$,

$G_{1 s m}\left(a_{k}, \psi_{k}\right)=\frac{1}{\Delta} \int_{0}^{l}\left\{G_{1 s}\left(a_{k}, x, \psi_{k}\right) \sum_{j=1} \Phi_{j}\left(x_{s}\right) \Phi_{j}(x)\right\} \Phi_{m}(x) d x \bar{\alpha}_{k m}^{2}\left(a_{k}, \psi_{k}\right)$ is a known function.

Below, we consider only differential equations that describe the laws of change of amplitude and frequency of torsional oscillations of an elastic body, i.e., the system of differential equations (12). Its right-hand sides are $2 \Pi$ periodic $\psi_{k}=\omega_{k}\left(a_{k}\right) t+\vartheta$ and $2 \Pi$ are periodic by the argument $\theta=\frac{2 \pi}{\tau} t$. Thus, for the considered system, it is necessary to consider two cases: $p \frac{2 \Pi}{\omega(a)} \approx q \tau$ and $p \frac{2 \Pi}{\omega(a)} \neq q \tau$ ( $p, q$ - mutually prime numbers). The first case will be called resonant. It occurs when there is a connection between the period in time of the natural $\frac{2 \Pi}{\omega(a)}$ and pulsed external perturbation in the form of rational correlation. In nonresonant cases, the phases of natural oscillations and pulsed external perturbation are not connected by rational correlation. As follows from the first equation of the system (21), the amplitude of oscillations is proportional to the small parameter $\mu$. This means that during natural or forced oscillations, the amplitude changes by the value of the order $\mu$. This is the basis for using the averaging apparatus [2] for differential equations (20) for the phases of natural oscillations and 
"reduced" pulse perturbation. Thus, in the nonresonant case, the main parameters of torsional oscillations of a nonlinear-elastic body are described by the correlations

$$
\begin{aligned}
\frac{d a_{k}}{d t}=-\frac{\mu}{8 \pi \Pi \omega_{k}\left(a_{k}\right) \rho \Delta} \int_{0}^{l} \int_{0}^{2 \Pi} \int_{0}^{2 \pi}\left\{\begin{array}{l}
F_{1 k}\left(a_{k}, x, \psi_{k}\right)+ \\
+\sum_{s=1} G_{1 s}\left(a_{k}, x, \psi_{k}\right) \cos \theta \delta\left(\frac{\theta}{\tau}-\frac{2(s-1) \pi}{\tau}\right) \\
\sum_{j=1} \Phi_{j}\left(x_{s}\right) \Phi_{j}(x)
\end{array}\right\} d x d \psi_{k} d \theta, \\
\Phi_{k}(x) s a\left(1, v+1, \psi_{k}\right)
\end{aligned}
$$

$\frac{d \psi_{k}}{d t}=\omega_{k}\left(a_{k}\right)-$

$$
\begin{gathered}
-\frac{\mu(v+2)}{16 \pi \prod a_{k} \omega_{k}\left(a_{k}\right) \rho \Delta} \int_{0}^{l} \int_{0}^{2 \Pi} \int_{0}^{2 \pi}\left\{\begin{array}{l}
F_{1 k}\left(a_{k}, x, \psi_{k}\right)+ \\
+\sum_{s=1} G_{1 s}\left(a_{k}, x, \psi_{k}\right) \cos \theta \delta\left(\frac{\theta}{\tau}-\frac{2(s-1) \pi}{\tau}\right) \\
\sum_{j=1} \Phi_{j}\left(x_{s}\right) \Phi_{j}(x)
\end{array}\right\} d x d \psi_{k} d \theta \\
\Phi_{k}(x) c a^{v+1}\left(v+1,1, \psi_{k}\right)
\end{gathered}
$$

If the properties of $\delta$ are functions are used, the system of differential equations (21) can be somewhat simplified, namely,

$$
\begin{aligned}
& \frac{d a_{k}}{d t}=-\frac{\mu}{4 \pi \Pi \omega_{k}\left(a_{k}\right) \rho \Delta} \int_{0}^{l} \int_{0}^{2 \Pi}\left\{\begin{array}{l}
F_{1 k}\left(a_{k}, x, \psi_{k}\right)+ \\
\left.+\sum_{s=1} G_{1 s}\left(a_{k}, x, \psi_{k}\right) \cos (2(s-1) \pi) \sum_{j=1} \Phi_{j}\left(x_{s}\right) \Phi_{j}(x)\right\}, \\
\Phi_{k}(x) s a\left(1, v+1, \psi_{k}\right) d x d \psi_{k}
\end{array}\right. \\
& \frac{d \psi_{k}}{d t}=\omega_{k}\left(a_{k}\right)-\frac{\mu(v+2)}{8 \pi \Pi a_{k} \omega_{k}\left(a_{k}\right) \rho \Delta} \int_{0}^{l} \int_{0}^{2 \Pi}\left\{\begin{array}{l}
F_{1 k}\left(a_{k}, x, \psi_{k}\right)+ \\
\left.+\sum_{s=1} G_{1 s}\left(a_{k}, x, \psi_{k}\right) \cos (2(s-1) \pi) \sum_{j=1} \Phi_{j}\left(x_{s}\right) \Phi_{j}(x)\right\} . \\
\Phi_{k}(x) c a^{v+1}\left(v+1,1, \psi_{k}\right) d x d \psi_{k}
\end{array}\right.
\end{aligned}
$$

The methods of research of nonlinear resonant torsional oscillations.

Much more challenging to study and, at the same time, more important from the practical side is the resonant case. It differs significantly from the resonant case of quasilinear oscillations of elastic bodies. The frequency of natural torsional oscillations of a nonlinear-elastic body depends on the amplitude of oscillations. Thus, resonance in the considered elastic body takes place under the condition that the amplitude of the single-frequency process approaches the value

$$
a^{*}=\left(\frac{2 p \Pi}{\alpha q \tau}\right)^{\frac{2}{v}}\left(\frac{l}{\lambda \Pi_{x}}\right)^{1+\frac{2}{v}}
$$

Below Fig. 2, Fig. 3 shows the dependence of the amplitude at which there is resonance for some parameters of the elastic body. 
a) $l=2, \tau=0.1,100<\alpha<1000$

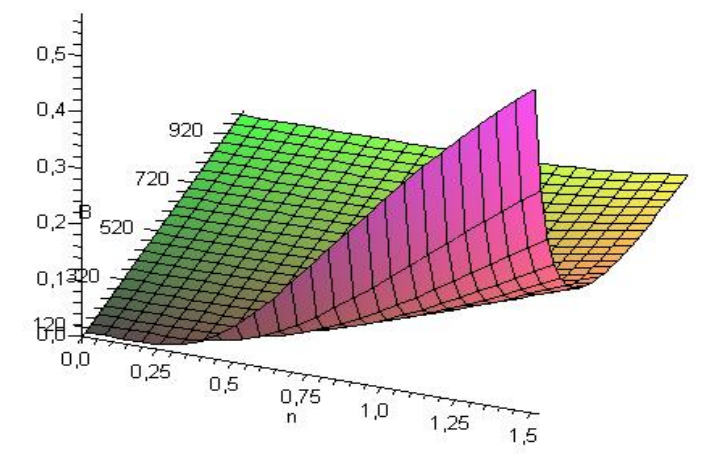

c) $l=2, \tau=0.05,100<\alpha<1000$

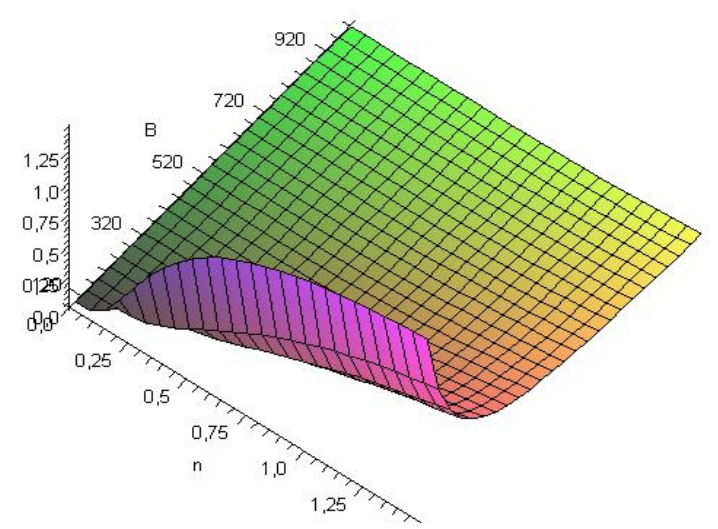

b) $l=2, \tau=0.08,100<\alpha<1000$

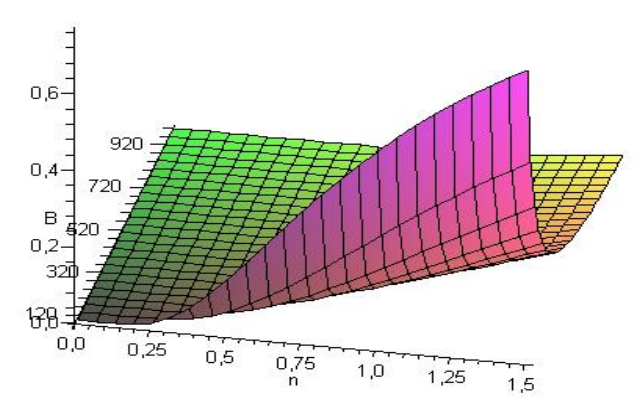

d) $l=2, \tau=0.001,100<\alpha<1000$

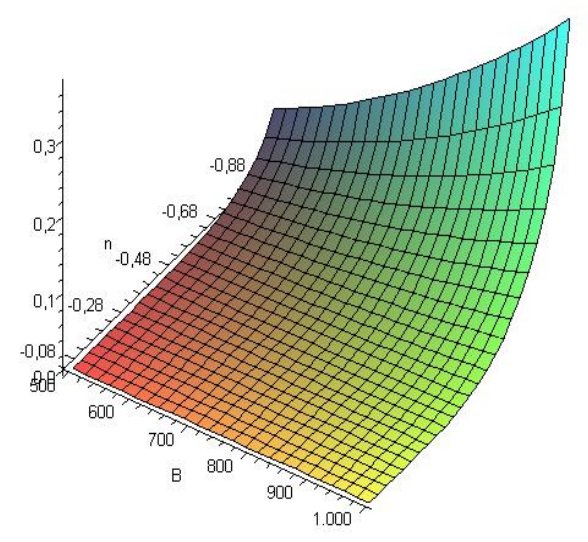

Figure 2: Dependence of the amplitude at which there is a resonance $a^{*}$ on the parameters of the system. $\alpha$ is a vert axis $a^{*}$
a) $\alpha=500, l=4$
b) $\alpha=500, l=4$
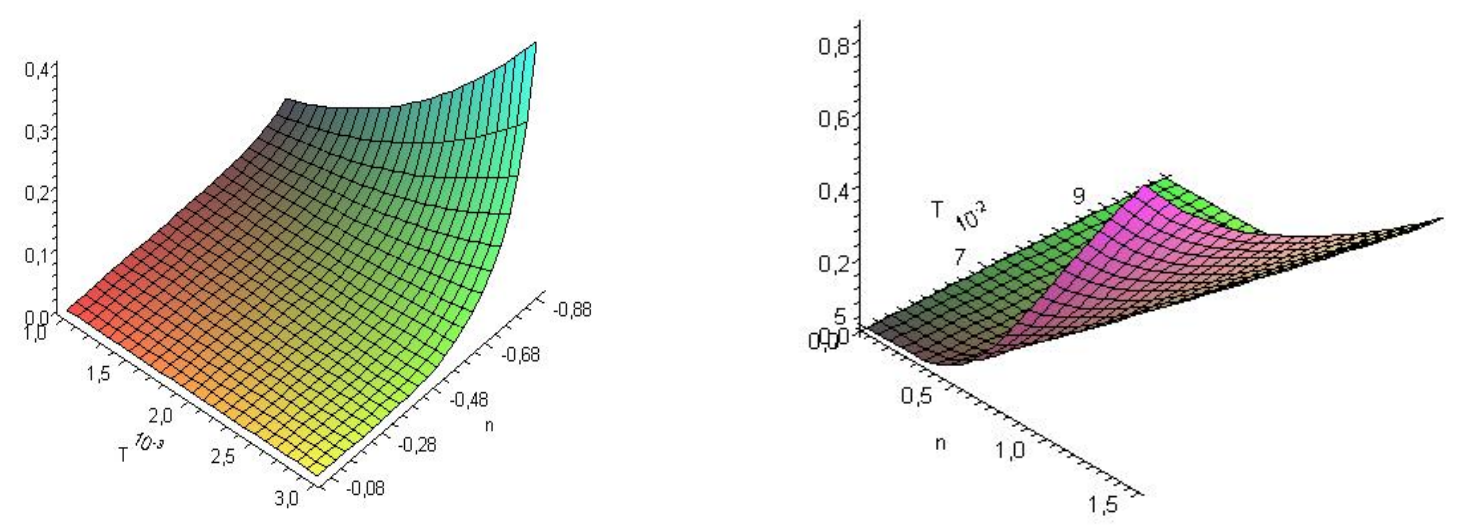

Figure 3: Dependence of the resonant amplitude on the nonlinearity parameter and the period of pulsed perturbation ( $\mathrm{n}-\mathrm{v}, \mathrm{T}-\tau$ vert axis $a^{*}$ in the Fig) 
The above graphical dependences show that in the case when the value of the nonlinearity parameter $v>0$ for smaller values of the parameter of "stiffness," $\alpha$ the resonance amplitude takes larger values, and the rate of increase of the resonance amplitude is more significant for more extensive parameters $v$, while for larger values of the pulse perturbation period resonance amplitude is more minor. For this case $-1<v<0$, a more considerable value of the parameter $\alpha$ responds to a more significant resonance amplitude value, and the resonance amplitude growth rate increases significantly when the parameter $v$ approaches -1 . In this case, the immense value of the pulse perturbation period responds to a larger resonance amplitude. The passing of resonance of the proposed elastic body, as for all nonlinear systems, depends significantly on the "phase difference" of natural oscillations and periodic perturbation $\gamma=q \frac{\pi}{\Pi} \psi-p \theta \rightarrow \psi=\frac{\Pi}{q \pi}(p \theta+\gamma)$. If we enter the specified parameter into the system of differential equations (23) and use for it the procedure of averaging by the "phase" of external pulse perturbation, we obtain for the case of main resonance $p=q=1$.

$$
\begin{aligned}
& \frac{d a_{k}}{d t}=-\frac{\mu}{4 \pi \Pi \omega_{k}\left(a_{k}\right) \rho \Delta}\left\{\int_{0}^{l} \int_{0}^{2 \Pi}\left[F_{1 k}\left(a_{k}, x, \psi_{k}\right) \Phi_{k}(x) s a\left(1, v+1, \psi_{k}\right) d x d \psi_{k}\right]+\right. \\
& -\frac{1}{\Delta} \int_{0}^{l} \int_{0}^{2 \pi} \sum_{s=1} G_{1 s}\left(a_{k}, x, \frac{\Pi}{\pi}(\theta+\gamma)\right) \cos \theta \delta\left(\frac{\theta}{\mu}-\frac{2(s-1) \pi}{\mu}\right\} \\
& \sum_{j=1} \Phi_{j}\left(x_{s}\right) \Phi_{j}(x) s a\left(1, v+1, \frac{\Pi}{\pi}(\theta+\gamma)\right) d x d \theta \\
& \frac{d \gamma}{d t}=\frac{\Pi}{\pi} \omega_{k}\left(a_{k}\right)-\frac{2 \pi}{\tau}- \\
& -\frac{\mu(v+2)}{4 \pi \Pi a_{k} \omega_{k}\left(a_{k}\right) \rho \Delta}\left\{\int_{0}^{l} \int_{0}^{2 \Pi}\left[F_{1 k}\left(a_{k}, x, \psi_{k}\right) \Phi_{k}(x) c a^{v+1}\left(v+1,1 \psi_{k}\right) d x d \psi_{k}\right]+\right. \\
& +\frac{1}{\Delta} \int_{0}^{l} \int_{0}^{2 \pi} \sum_{s=1} G_{1 s}\left(a_{k}, x, \frac{\Pi}{\pi}(\theta+\gamma)\right) \cos \theta \delta\left(\frac{\theta}{\mu}-\frac{2(s-1) \pi}{\mu}\right. \\
& \sum_{j=1} \Phi_{j}\left(x_{s}\right) \Phi_{j}(x) c a^{v+1}\left(1, v+1, \frac{\Pi}{\pi}(\theta+\gamma)\right) d x d \theta
\end{aligned}
$$

Since the resonance phenomenon occurs when the amplitude of oscillations approaches the value $a_{k}^{*}$ during the numerical simulation of the obtained system of differential equations (25), the following approaches are considered:

- for the resonance case, the value $\frac{\Pi}{\pi} \omega_{k}\left(a_{k}\right)-\frac{2 \pi}{\tau}$ in the second formula of dependences (25) can

be replaced by the following:

$$
\frac{d \omega_{k}\left(a_{k}\right)}{d a_{k} \mid a_{k}=a_{k}^{*}}\left(a_{k}-a_{k}^{*}\right)
$$
as it should be small; 
For some reasons, it is known, for example, that the frequency (period) of pulse perturbation is a slowly

varying function of time

$$
\left(\frac{2 \pi}{\tau}=\frac{\Pi}{\pi} \omega_{k}\left(a_{k}^{*}\right)+\varepsilon \eta(t), \eta(t)\right. \text { is a known function); then, the value }
$$

$\frac{\Pi}{\pi} \omega_{k}\left(a_{k}\right)-\frac{2 \pi}{\tau}$ in the second equation of correspondence (25) can be replaced by the value $\varepsilon \eta(t)$.

Notes:

- The paper considers that the studied body has a limited size, so the amplitude and the frequency of the process change only with time. The case of "long systems" $[16,17]$ can be the subject of separate studies.

- For the initial conditions, they must ensure the existence of oscillating motion in an undisturbed system.

- The initial value of the amplitude for a system of differential equations (25) in both of the above cases should be close to $a_{k}^{*}$.

- The boundary value problems considered in this paper also describe the relative torsional oscillations of an elastic body under the action of a system of impulse moments, provided that the portable angular velocity of rotation of the body is a constant value.

\section{Discussion}

A crucial case of the dynamics of the elastic body specified in paragraph 4 of the notes is when the pulsing momentum acts on the elastic body at a fixed point and is repeated during the complete rotation of the body $N$ times at regular intervals. Differential equation of relative elastic torsional oscillations of such body under the conditions that:

- the force of resistance is proportional to the speed of the relative torsion angle;

- elastic properties are described by dependence $\sigma=k \varepsilon^{v+1}+\mu \beta \varepsilon^{3}$.

- takes the form

$$
\phi_{t t}-\alpha^{2}\left(\phi_{x}\right)^{v} \phi_{x x}=-\frac{\mu}{\rho}\left\{\beta_{1} \phi_{t}+\beta_{2}\left(\phi_{x}\right)^{2} \phi_{x x}-M \delta\left(x-x_{0}\right) \sum_{s=1} \delta\left(t-s \frac{2 \pi}{N \Omega}\right)\right\}
$$

where $\bar{\beta}_{1}, \bar{\beta}_{2}$ are known constants, $\Omega$ is the angular velocity of rotation of the body, $\frac{2 \pi}{N \Omega}$ is the period of action of pulse momentum, the value of which is equal to $\mu M$, and $x_{0}$ is the point of application of the moment. In this case, nonresonant correlations (22) for the case of boundary conditions (2) take the form of

$$
\begin{aligned}
& \frac{d a_{k}}{d t}=-\mu \bar{\beta}_{1} a_{k}+\frac{\mu M}{4 \pi \Pi \omega_{k}\left(a_{k}\right) \rho \Delta^{2}} \sum_{s=1} \int_{0}^{l} \int_{0}^{2 \Pi} \int_{0}^{2 \pi} \cos \theta\left(\delta\left(\frac{N \theta \Omega}{2 \pi}-s \frac{2 \pi}{N \Omega}\right)\right)
\end{aligned}
$$

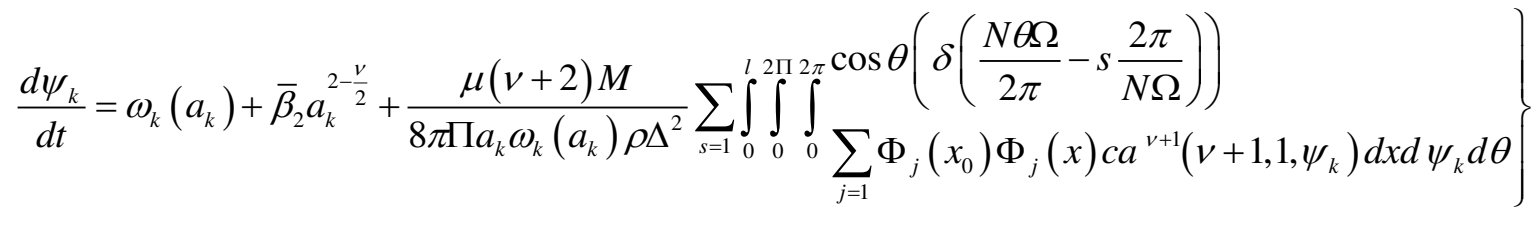


where $\bar{\beta}_{1}, \bar{\beta}_{2}$ are the known constants.

From the properties of periodic Ateb functions, the independence of the phases of natural and forced (pulse) oscillations, it follows that the integrals in the right parts of the dependences (27) are zero

because $\int_{0}^{2 \Pi} s a\left(1, v+1, \psi_{k}\right) d \psi_{k}=\int_{0}^{2 \Pi} c a^{v+1}\left(1, v+1, \psi_{k}\right) d \psi_{k}=0$, and therefore nonresonant torsional oscillations, in this case, are described by differential equations

$$
\frac{d a_{k}}{d t}=-\mu \bar{\beta}_{1} a_{k}, \frac{d \psi_{k}}{d t}=\omega_{k}\left(a_{k}\right)+\bar{\beta}_{2} a,^{2-\frac{v}{2}}
$$

Thus, relation (28) confirms that in the case of periodic impulse perturbation, which does not coincide with the period of natural torsional oscillations, the dynamic process is determined only by dissipative and nonlinear elastic forces. For the resonant oscillations of the above case of torsional oscillations of an elastic body, the differential equations that describe the principal resonance in the first mode of oscillations under boundary conditions (3) take the form

$$
\begin{aligned}
& \frac{d a}{d t}=-\mu \bar{\beta}_{1} a_{k}-\frac{\mu M}{4 \pi \Pi \omega(a) \rho \Delta^{2}} s a\left(1, v+1, \frac{\Pi}{\pi} \gamma\right) \sum_{j=1} s a\left(1, \frac{1}{v+1}, \frac{j}{l} \Pi_{x} x_{0}\right), \\
& \frac{d \gamma}{d t}=\frac{d \omega(a)}{d a}{ }_{\mid a=a *}\left(a-a^{*}\right)+\bar{\beta}_{2} a,^{2-\frac{v}{2}}+\frac{\mu(v+2) M}{8 \pi \Pi a \omega(a) \rho \Delta^{2}} c a^{v+1}\left(v+1,1, \frac{\Pi}{\pi} \gamma\right) \sum_{j=1} s a\left(1, \frac{1}{v+1}, \frac{j}{l} \Pi_{x} x_{0}\right)
\end{aligned}
$$

Below in Fig. 4, following the differential equations (29), for the resonance case, the laws of change of the amplitude of oscillations during the transition through the main resonance are presented.
a) $x_{0}=1$
b) $x_{0}=0.8$
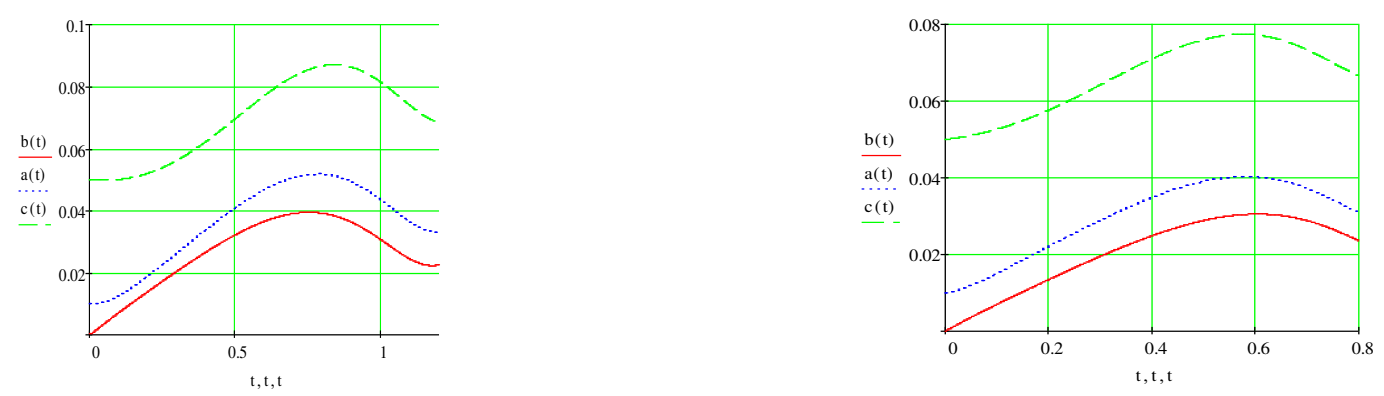

$l=1, v=0.22$ green; $v=0.44$ red $; v=-0.22$ blue are for all below

c) $x_{0}=0.5$

d) $x_{0}=0.4$
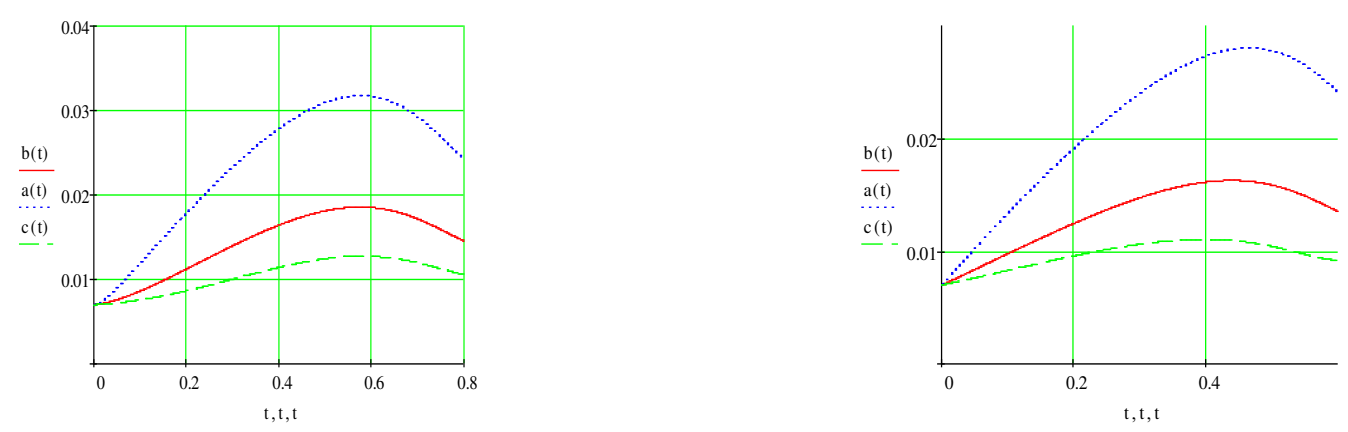
f) $x_{0}=0.3$

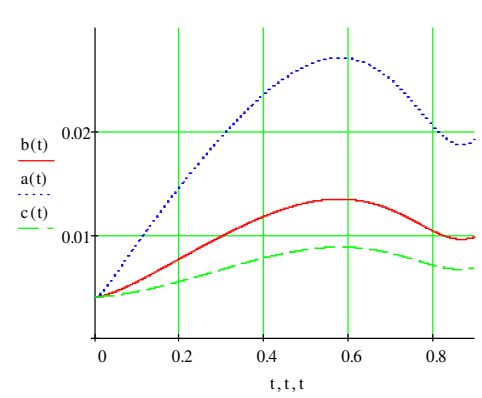

g) $x_{0}=0.2$

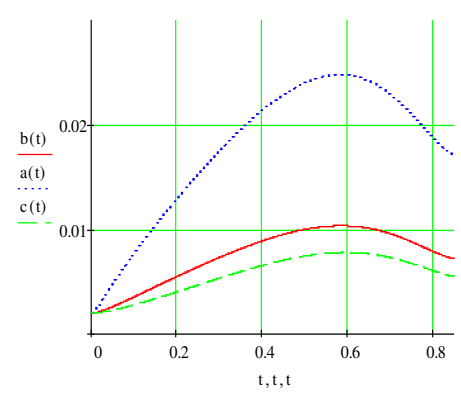

Figure 4: The laws of the change of the amplitude of torsional oscillations at transition through the primary resonance at various points of application of pulse perturbation.

where a) $x_{0}=1, l=2, \alpha=1 \cdot 10^{3},-$ curve $1,-$ curve $2,-$ curve 3 ;

b) $x_{0}=0.8, l=2, \alpha=1 \cdot 10^{3}, v=0.1,-$ curve $1,-$ curve $2,-$ curve 3 ;

c) $x_{0}=0.5, l=1, \alpha=1 \cdot 10^{3}, v=0.22$, - curve $1,-$ curve $2,-$ curve 3 ;

d) $x_{0}=0.4, l=1, \alpha=1 \cdot 10^{3}, v=0.22,-$ curve $1,-$ curve $2,-$ curve 3 ;

f) $x_{0}=0.3, l=1, \alpha=1 \cdot 10^{3}, v=0.22$, - curve $1,-$ curve $2, v=-0.22,-$ curve 3 ;

g) $x_{0}=0.2, l=1, \alpha=1 \cdot 10^{3}, v=0.22,-$ curve $1, v=0.44,-$ curve $2, v=-0.22,-$ curve 3 ;

Theoretical results and graphical dependences based on their resonant torsional oscillations of a nonlinearly elastic body under the action of a periodic system of pulse momentum applied to one geometric cross-section of the body under boundary conditions (3) show in particular that the amplitude of resonance transition is larger:

- for larger values of nonlinearity parameter ${ }^{v}$ (at all other invariant basic body parameters and $v>0$ );

- In the case of the action of momentum closer to the middle of the body for boundary conditions (2) and (3) and for boundary conditions (4), the opposite is true.

\section{Conclusions}

The research method of the influence of impulse moments on torsional oscillations of strongly nonlinear elastic bodies is developed in this work. Analysis of the obtained theoretical and constructed graphical results shows that their periodic action on the body of impulse moments in the body occurs as nonresonant and resonant oscillations. Resonant oscillations occur when the amplitude of torsional oscillations approaches a specific value - the amplitude of resonance. The amplitude of resonance in a case when the parameter of nonlinearity is $v>0$ for:

- smaller values of the parameter of "stiffness" $\alpha$ takes the more considerable value, and its growth rate is more significant for larger values of the parameter

- For larger values of the period of pulsed perturbation, the resonance amplitude is more minor. In this case, $-1<v<0$ a more considerable value $\alpha$ corresponds to the immense value of amplitude resonance, and the growth rate of the amplitude increases significantly while approaching $v$ to -1 . Suppose the nonlinearly elastic body is only under the periodic system of impulse moment forces and 
dissipative forces to avoid simultaneous resonance oscillations. In that case, the amplitude of the initial perturbation must be slightly smaller than the amplitude of resonance. Otherwise, a phenomenon similar to beating in linear systems will occur. For the amplitude of the passage through the resonance under the action on the body of the periodic system of pulse moments of the same magnitude in the same cross-section of the body, the amplitude of the transition through the resonance is greater:

- for larger values of the nonlinearity parameter $v$ (for all other invariant basic body parameters and $v>0$ );

- in the case of an impulse moment closer to the middle of the body and for boundary conditions (3). The reliability of the work results is confirmed by the fact that in extreme cases (

$v=0, \mathrm{~h}_{s}\left(\phi, \phi_{t}, \phi_{x}, \phi_{x x}\right)=0$ ), we know from the literature $[18,19,20]$. The question of the flow and the conditions of existence of a single-frequency process, depending on the initial conditions of the system, can be the subject of separate studies.

Practical application of the obtained results.

The results obtained in this work can be the basis for the choice of operating parameters of machine elements of mechanisms that are exposed to instantaneous moments to avoid resonant phenomena, in particular, rotors of multistage compressors of gas turbine engines [18]; research of dynamic processes in machines with flexible high-speed rotors to justify the choice of the most stable frequency of its rotation.

Conflicts of Interest: The authors declare that there are no conflicts of interest.

\section{References}

1. Babakov, "Theory of oscillations," Moscow, Russia: Nauka, pp. 1-560, 1965.

2. Y. Mitropolskii and B. Moseenkov, "Asymptotic solutions of partial differential equations," Kyiv, Ukraine: Vyshcha Shkola, pp. 1-596, 1976.

3. G. Pisarenko, O. Kvitka and E. Umansky, "Resistance of materials," Kyiv, Ukraine: Vyshcha shkola, pp. 1-654, 2004.

4. Yu. Mitropolsky and B. Sokil, "On the application of Ateb-functions for constructing an asymptotic solution of a perturbed nonlinear Klein-Gordon equation," Ukrainian Mathematical Journal, vol. 50, no. 5, pp. 665-670, 1998.

5. B. Sokil, "Construction of asymptotic solutions of certain boundary-value problems for the no autonomous wave equation," Journal of Mathematical Sciences, vol. 96 (1), pp. 2878-2882, 1999.

6. V. Maslov, "Asymptotic methods and perturbation theory," Moscow, Russia: Nauka, pp. 1-312, 1988.

7. P. Senik, "Inversion of the incomplete beta function," Ukrainian Mathematical Journal, vol. 21, no.3, pp. 271-278, 1969.

8. M. Nazarkevych, "Investigation of Beta- and Ateb-function dependencies," Bulletin of the Lviv Polytechnic National University. Series: Computer Science and Information Technology, vol.732, pp. 207-216, 2012.

9. V. Olshansky, V. Burlaka, M. Vlipchenko and O. Malets, "On oscillations of an oscillator with cubic-nonlinear stiffness," Bulletin of National Technical University "Kharkiv Polytechnic Institute", Series: Dynamics and strength machines, vol. 39, pp. 177-184, 2017.

10. L. Cveticanin, "Period of vibration of axially vibrating truly non-linear rod," Journal of Sound and Vibration, vol. 374, pp.199-210, 2016. 
11. O. Gendelman and A. Vakakis, "Transitions from localization to nonlocalization in strongly nonlinear damped oscillators," Chaos, Solitons and Fractals, vol. 11, no. 10, pp.1535-1542, 2000.

12. E. Weisstein, Delta Function. [Online]. Available: https://mathworld.wolfram.com/DeltaFunction.html

13. Andrukhiv, B. Sokil, M. Sokil, Yu. Chahan and N. Huzyk, "Methodology of investigation of the influence of the explosion on the elements of protective structures," Military-Technical Collection. National Academy of Land Forces, vol. 22, pp. 32-37, 2020.

14. Sokil and A. Barvinskii, "An asymptotic expansion for a class of nonlinear differential equations," Ukrainian Mathematical Journal, vol. 32, no. 5, pp. 465-468, 1980.

15. Yu. Mitropolsky and O. Limarchenko, "On the question of asymptotic approximations for slow wave processes in nonlinear dispersing media," Ukrainian Mathematical Journal, vol. 59, no. 3, pp. 357-371, 1998.

16. Yu. Mitropolsky, "On the construction of the asymptotic solution of the perturbed Breserton equation," Ukrainian Mathematical Journal, vol. 59, no. 1, pp. 58-71, 1998.

17. K. Velten, "Mathematical modeling and simulation: introduction for scientists and engineers," Wiley-VCH Verlag GmbH \& Co. KGaA, Weinheim, pp. 1-362, 2009.

18. Andrukhiv, M. Sokil, S. Fedushko, Y. Syerov, Y. Kalambet et al., "Methodology for Increasing the Efficiency of Dynamic Process Calculations in Elastic Elements of Complex Engineering Constructions," Electronics, vol. 10 (1), no. 40, 2021. [Online]. Available: https://doi.org/10.3390/electronics10010040

19. N. Pirogova and P. Taranenko, "Calculation-experimental analysis of inner values and critical frequencies and forms of high-speed rotor of a microgas turbine installations," Mechanical Engineering, vol. 15. pp. 37-47, 2015. 